\title{
Increased serum interleukin-6 levels in patients with hidradenitis suppurativa
}

\author{
Haoxiang $\mathrm{Xu}^{1,2}$, Xuemin $\mathrm{Xiao}^{3}$, Yanyan $\mathrm{He}^{1,2}$, Xiaofeng Zhang ${ }^{1,2}$, Chengrang $\mathrm{Li}^{1,2}$, Qiuxia Mao ${ }^{1,2}$, Xinfeng $\mathrm{Wu}^{1,2}$, \\ Baoxi Wang 2,4
}

${ }^{1}$ Institute of Dermatology, Chinese Academy of Medical Sciences, Jiangsu, China ${ }^{2}$ Jiangsu Key Laboratory of Molecular Biology for Skin Diseases and STIs, Jiangsu, China ${ }^{3}$ Department of Dermatology, Union Hospital, Fujian Medical University, Fujian, China ${ }^{4}$ Department of Dermatology, Institute of Plastic Surgery, Chinese Academy of Medical Sciences, Beijing, China

Adv Dermatol Allergol 2017; XXXIV (1): 82-84 DOI: https://doi.org/10.5114/ada.2017.65626

Hidradenitis suppurativa (HS), also known as acne inversa, is a chronic, recurrent, and inflammatory skin disease. It has a complex etiology and is associated with genetic factors, sex hormones, bacterial infection, and immune dysfunctions [1]. Studies have indicated that a variety of cytokines such as tumor necrosis factor $\alpha$ (TNF- $\alpha$ ), interleukin (IL)-1 $\beta$, and IL-10 are involved in the pathogenesis of HS [2, 3]. Interleukin-6 is a cytokine with a molecular weight between 21 and 30 kDa. Abnormal upregulation of IL-6 has been proved to play pathological roles in many inflammatory diseases such as rheumatoid arthritis and Crohn's disease [4], and IL-6 is considered as an inflammatory marker [5, 6]. Recently, HS has been referred to be systemic auto-inflammatory disease [7].

Alterations of IL-6 levels in the serum of HS patients have not been reported and this study was aimed to measure the serum IL-6 levels in patients with HS.

This study was approved by the Ethics Committee of our hospital and conducted according the Declaration of Helsinki Principles, and all study subjects signed an informed consent form. Serum samples were obtained from 44 Chinese Han patients (36 men and 8 women) who met the diagnostic criteria for HS [1]. The patients were classified according to the Hurley stage [1]. The study participants included 18 cases of Hurley stage I patients, 12 cases of Hurley stage II patients, and 14 cases of Hurley stage III patients (Table 1). The differences in sex, age, and disease history among the three groups were not significant $(p>0.05)$. The healthy control group was comprised of 13 healthy volunteers without any abnormal clinical or laboratory findings (Table 1). The differences in sex and age between healthy control group and patient groups showed no significance $(p>0.05)$.

Morning fasting peripheral venous blood samples $(4 \mathrm{ml})$ were collected from study subjects. After settling for $20 \mathrm{~min}$, the samples were centrifuged $(1000 \times \mathrm{g}$, $10 \mathrm{~min}, 4^{\circ} \mathrm{C}$ ) and placed in a $-80^{\circ} \mathrm{C}$ freezer for subsequent use. IL-6 ELISA reagent kit was purchased from eBioscience (San Diego, CA, USA; catalogue no. BMS213/2). The procedure was performed according to the kit instruction, and results were calculated when absorbance of each well was measured at $450 \mathrm{~nm}$ with a spectrophotometer (BioTek ELX 800, Winooski, VT, USA).

Statistical analysis was performed using the one-way analysis of variance for comparison between different groups, and the Mann-Whitney $U$ test for comparison of two independent samples. $P$-value $<0.05$ was considered statistically significant.

Table 1. Characteristics of healthy control and patient groups

\begin{tabular}{lcccccc}
\hline Group & \multicolumn{3}{c}{ Number } & Age (range) [years] & $\begin{array}{c}\text { Disease history (range) } \\
\text { [years] }\end{array}$ \\
\cline { 2 - 5 } Healthy control & Total & Male & Female & & $45.2 \pm 19.8(20-77)$ & - \\
\hline Hurley I & 13 & 11 & 2 & $41.5 \pm 19.2(15-74)$ & $25.6 \pm 12.8(1-40)$ \\
\hline Hurley II & 18 & 14 & 2 & $40.3 \pm 20.6(17-82)$ & $27.8 \pm 13.3(1-42)$ \\
\hline Hurley III & 12 & 12 & 2 & $44.2 \pm 23.7(17-79)$ & $26.9 \pm 15.5(2-46)$ \\
\hline
\end{tabular}

Address for correspondence: Xinfeng Wu, Jiangsu Key Laboratory of Molecular Biology for Skin Diseases and STIs, Jiangsu, China, phone: 86-13675109778, fax: 86-25-85478035, e-mail: wuxinfengdr@163.com; Baoxi Wang, Jiangsu Key Laboratory of Molecular Biology for Skin Diseases and STIs, Jiangsu, China, phone: 86-13815441336, fax: 86-10-88771611, e-mail: wangbx@ncstdlc.org Received: 23.11.2015, accepted: 11.04.2016. 
Compared to the IL-6 levels in the healthy control group, the serum IL- 6 levels in Hurley stage II and III patients were significantly increased (Figure 1). Then the patients were grouped by sex, disease history, lesion area, the presence of diabetes mellitus, and smoking. The IL-6 levels of patients with lesions covering $<5 \%$ of their body surface were significantly lower than those with lesions covering $>5 \%$ of their body surface, while the differences between all other groups were not significant (Table 2).

Interleukin-6 is secreted by macrophages, neutrophils, lymphocytes, keratinocytes, and fibroblasts. It mainly participates in B lymphocytes differentiation, T lymphocytes activation, antibody production, increasing acute phase proteins, and enhancing immunity against infections. In an inflammatory reaction, IL-6 not only has an anti-inflammation function but could also exhibit pro-inflammatory activity [8].

Hidradenitis suppurativa is considered to be an auto-inflammatory disease [7]. The innate and adaptive immune responses in HS patients have been altered, and many cytokines are associated with HS development [9]. At present, there were only a few reports on the association between IL- 6 and HS, and the results were not consistent. Bechara et al. reported that, compared to the non-lesion area, IL-6 mRNA expression was increased in lesions of HS patients [10]. Van der Zee et al. cultured HS skin lesions to determine the IL-6 levels [11]. They showed that the IL- 6 levels were significantly increased and were above the upper detection limit of the reagent kits. However, Dréno et al. revealed that the IL-6 levels in HS skin lesions were lower than that in non-lesioned skin [12]. Giamarellos-Bourboulis et al. also showed that in vitro cultured monocytes from HS patients exhibited an impaired ability to secrete IL-6, which did not concur with ours [9]. One possible reason for this discrepancy is that many cell types produce IL-6, but they only studied the IL-6 secretion of monocytes. Histological findings

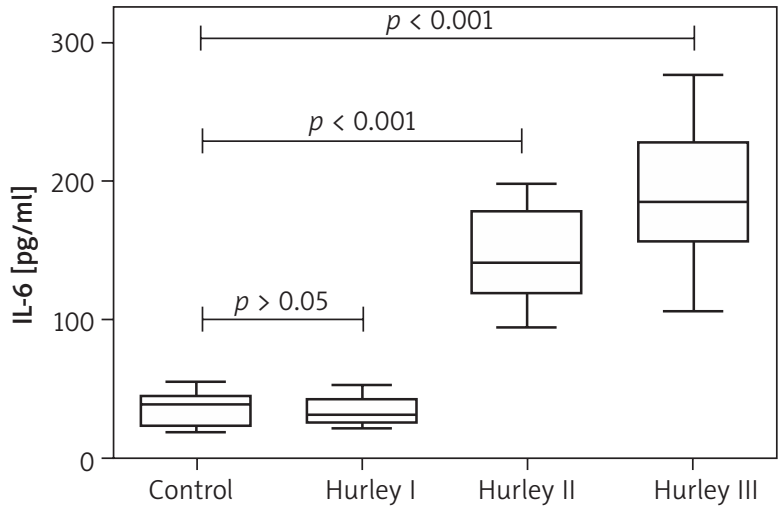

Figure 1. Comparison of serum IL-6 levels between healthy controls and HS patients with different degrees of disease severity. Serum IL-6 levels in a group of Hurley stage I, II, and III patients were $33.4 \pm 8.9 \mathrm{pg} / \mathrm{ml}$ (range: $22.3-52.8 \mathrm{pg}$ / $\mathrm{ml}$ ), $145.6 \pm 33.6 \mathrm{pg} / \mathrm{ml}$ (range: $94.6-198.0 \mathrm{pg} / \mathrm{ml}$ ), and 191.4 $\pm 48.1 \mathrm{pg} / \mathrm{ml}$ (range: $106.4-301.1 \mathrm{pg} / \mathrm{ml}$ ), respectively. Compared to IL-6 levels in the healthy control group (34.9 $\pm 11.4 \mathrm{pg} / \mathrm{ml}$, range: $18.8-55.5 \mathrm{pg} / \mathrm{ml}$ ), the serum IL-6 levels in Hurley stage II and III patients were significantly increased, and the difference was statistically significant ( $p$-values of both $<0.001)$

of lesions in HS patients typically showed epithelioid granuloma changes, and IL- 6 is involved in the formation of chronic granulomatous skin conditions [13]. Elevated serum IL-6 levels in Hurley II and III patients not only participate in maintenance of inflammation but also might promote formation of granulomas in lesions. Intriguingly, HS always coexists with other inflammatory diseases of which IL-6 also takes part in the development, including pyoderma gangrenosum, inflammatory bowel disease, arthritis and spondyloarthropathy, implying that they share similar immune-pathogenetic pathways $[4,14]$.

Table 2. Comparison of serum IL-6 levels in different groups of HS patients

\begin{tabular}{|c|c|c|c|c|}
\hline Parameter & & Number of cases & Serum IL-6 level $(\bar{x} \pm s$ D) & $P$-value ${ }^{\#}$ \\
\hline \multirow[t]{2}{*}{ Gender } & Male & 36 & $120.0 \pm 48.0$ & 0.843 \\
\hline & Female & 8 & $103.3 \pm 37.4$ & \\
\hline \multirow{2}{*}{$\begin{array}{l}\text { Disease history } \\
\text { [years] }\end{array}$} & $<10$ & 15 & $118.6 \pm 48.0$ & 0.99 \\
\hline & $>10$ & 29 & $110.6 \pm 43.3$ & \\
\hline \multirow[t]{2}{*}{ Skin lesion area } & $<5 \%$ & 34 & $93.8 \pm 62.1$ & 0.004 \\
\hline & $>5 \%$ & 10 & $183.9 \pm 50.1$ & \\
\hline \multirow[t]{2}{*}{ Diabetes mellitus } & - & 39 & $110.5 \pm 37.8$ & 0.698 \\
\hline & + & 5 & $130.2 \pm 41.4$ & \\
\hline \multirow[t]{2}{*}{ Smoking } & - & 28 & $110.1 \pm 38.1$ & 0.807 \\
\hline & + & 16 & $120.5 \pm 28.5$ & \\
\hline
\end{tabular}

\#Mann-Whitney U test for comparison of two independent samples was used. *According to factors including sex, disease history, lesion area, the presence of diabetes mellitus, and smoking, serum IL-6 levels of the healthy control group showed no statistical significance. 
In this study, we also showed that IL-6 levels of patients with lesions covering $>5 \%$ of body surface were significantly higher than those with lesions covering $<5 \%$ of body surface. This result suggested that the lesion area affects immune-related cells and/or the systemic immune status of HS patients. Notably, IL-6 has a broad effect on cells of the immune system and nonimmune system, but it can also be activated by other factors in the inflammatory reaction [15]; therefore, it remains undetermined whether IL-6 could be used as a new biomarker to scoring HS severity or monitoring prognosis. The limitation of this study was that the number of female cases was not adequate, and a larger number of enrolled HS patients would improve the credibility of our findings.

In general, this study showed that the serum IL-6 levels were increased in Hurley II and III HS patients, suggesting that IL-6 plays a role in the development of HS. Meanwhile, the exact mechanism of IL- 6 participation in the development of HS awaits further studies.

\section{Acknowledgments}

Haoxiang Xu and Xuemin Xiao contributed equally to the work.

This work was funded by grants from National Nature Science Foundation of China (Grant No. 81101207, 81472905, 81472872), Research Fund for the Doctoral Program of Higher Education (20111106120052), Fundamental Research Funds for the Central Universities \& the Beijing Union Medical College Youth Research Fund (3332013056), and Janssen Research Fund (JRCC2011derm-01).

\section{Conflict of interest}

The authors declare no conflict of interest.

\section{References}

1. Jemec GB. Clinical Practice. Hidradenitis suppurativa. N Engl J Med 2012; 366: 158-64.

2. Kelly G, Sweeney CM, Tobin AM, Kirby B. Hidradenitis suppurativa: the role of immune dysregulation. Int J Dermatol 2014; 53: 1186-96.

3. Matusiak L, Bieniek A, Szepietowski JC. Increased serum tumour necrosis factor-alpha in hidradenitis suppurativa patients: is there a basis for treatment with anti-tumour necrosis factor-alpha agents? Acta Derm Venereol 2009; 89: 601-3.

4. Kishimoto T. IL-6: from its discovery to clinical applications. Int Immunol 2010; 22: 347-52.

5. Rincon M, Irvin CG. Role of IL-6 in asthma and other inflammatory pulmonary diseases. Int J Biol Sci 2012; 8: 1281-90.

6. Seyedmirzaee S, Hayatbakhsh MM, Ahmadi B, et al. Serum immune biomarkers in irritable bowel syndrome. Clin Res Hepatol Gastroenterol 2016; 40: 631-7.

7. Vekic DA, Frew JW, Woods J, Cains GD. Adopting the orphan: the importance of recognising hidradenitis suppurativa as a systemic auto-inflammatory disease. Australas I Dermatol 2016; 57: 69-70.

8. Ingram JR, Woo PN, Chua SL, et al. Interventions for hidradenitis suppurativa: a cochrane systematic review incorporating GRADE assessment of evidence quality. Br I Dermatol 2016; 174: 970-8.

9. Giamarellos-Bourboulis EJ, Antonopoulou A, Petropoulou C, et al. Altered innate and adaptive immune responses in patients with hidradenitis suppurativa. Br I Dermatol 2007; 156: 51-6.

10. Bechara FG, Sand M, Skrygan M, et al. Acne inversa: evaluating antimicrobial peptides and proteins. Ann Dermatol 2012; 24: 393-7.

11. van der Zee HH, de Ruiter L, van den Broecke DG, et al. Elevated levels of tumour necrosis factor (TNF)-alpha, interleukin (IL)-1beta and IL-10 in hidradenitis suppurativa skin: a rationale for targeting TNF-alpha and IL-1beta. Br J Dermatol 2011; 164: 1292-8.

12. Dreno B, Khammari A, Brocard A, et al. Hidradenitis suppurativa: the role of deficient cutaneous innate immunity. Arch Dermatol 2012; 148: 182-6.

13. Ahmed AA, Nordlind K, Schultzberg M, Liden S. Interleukin-1 alpha- and beta-, interleukin-6- and tumour necrosis factoralpha-like immunoreactivities in chronic granulomatous skin conditions. Acta Derm Venereol 1994; 74: 435-40.

14. Kohorst JJ, Kimball AB, Davis MD. Systemic associations of hidradenitis suppurativa. I Am Acad Dermatol 2015; 73: S27-35.

15. Hunter CA, Jones SA. IL- 6 as a keystone cytokine in health and disease. Nat Immunol 2015; 16: 448-57. 